\title{
Don't gamble the COVID-19 response on ecological hypotheses
}

To the Editor - Araújo et al. have published a response to our piece 'Species distribution models are inappropriate for COVID-19'1 entitled 'Ecological and epidemiological models are both useful for SARS-CoV-2', in which they defend the idea that ecological models are likely to identify the signature of climate drivers in the $R_{0}$ of COVID-19 transmission.

Nevertheless, we are disappointed to see no attempt to provide any evidence supporting such a perspective. Araújo et al. state that "the point made by Carlson et al. was that top-down correlative models are inappropriate, whereas bottom-up epidemiological models are fine." ${ }^{2}$ This framing moves the goalposts and fully misrepresents our piece. We wrote that one highly specialized correlative tool of many (species distribution modelling) is inappropriate for one specific use case (forecasting a directly transmitted virus), with the aim of preventing incorrect work from being published and negatively impacting policy. We stand by that warning, as no new evidence about SARS-CoV-2 changes the limitations we identified.

Unfortunately, the authors' response includes no explicit discussion of species distribution models, any actual evidence of a climate-transmission link for SARS-CoV-2, or any direct engagement with the microbiological evidence previously outlined. Instead, the authors have proposed a number of new immunological and ecological hypotheses about how climate could drive COVID-19 transmission, some with tenuous mechanisms not currently supported by medical science (for example, perhaps climate creates geographic differences in innate immune responses to a novel pathogen, or changes the duration of infection?). None are testable by broad, correlative ecological methods; although COVID-19 seems to have spread globally unencumbered by climate $^{3}$, ecological modelling efforts that continue to try and dig up a climate 'signal' (heavily confounded by testing, reporting and outbreak timing) will rely on and further perpetuate these unsubstantiated claims.

At such a fragile moment for scientific accuracy and transparency, we are disappointed to see ecologists meet virological data with speculation that could easily gain a life of its own and inform sensitive policy decisions ${ }^{4}$. In their response, the authors explain that ecologists and epidemiologists should work in tandem, drawing on different lines of evidence and reasoning to form opinions that can best guide policy. As a team doing exactly that, including several experts on species distribution modelling and an eco-epidemiologist working on the
COVID-19 response, we suggest that they stop speculating and start listening.

Colin J. Carlson (D1 1⿴囗十, Joseph D. Chipperfield ${ }^{2}$, Blas M. Benito ${ }^{3}$, Richard J. Telford (iD ${ }^{4}$ and Robert B. O'Hara (iD 5

${ }^{1}$ Center for Global Health Science and Security, Georgetown University Medical Center, Washington, DC, USA. ${ }^{2}$ Norwegian Institute for Nature Research, Bergen, Norway. ${ }^{3}$ Department of Ecology \& Multidisciplinary Institute for Environment Studies "Ramon Margalef", University of Alicante, Alicante, Spain. ${ }^{4}$ Department of Biological Sciences, University of Bergen and Bjerknes Centre for Climate Research, Bergen, Norway. ${ }^{5}$ Department of Mathematical Sciences and Centre for Biodiversity Dynamics, Norwegian University of Science and Technology, Trondheim, Norway.

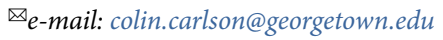

Published online: 29 July 2020

https://doi.org/10.1038/s41559-020-1279-2

References

1. Carlson, C. J., Chipperfield, J. D., Benito, B. M., Telford, R. J. \& O'Hara, R. B. Nat. Ecol. Evol. 4, 770-771 (2020).

2. Araújo, M. B., Mestre, F. \& Naimi, B. Nat. Ecol. Evol. https://doi. org/10.1038/s41559-020-1246-y (2020).

3. O'Reilly, K. M. et al. Lancet Planet. Health 4, el72 (2020).

4. Chipperfield, J. D., Benito, B. M., O'Hara, R., Telford, R. J. \& Carlson, C. J. Preprint at EcoEvoRxiv https://doi.org/10.32942/osf. io/mr6pn (2020).

Competing interests

The authors declare no competing interests. 